\title{
Exact inference on scaling parameters in norm and antinorm contoured sample distributions
}

\author{
Wolf-Dieter Richter
}

Correspondence:

wolf-dieter.richter@uni-rostock.de Department of Mathematics, University of Rostock, Rostock, Germany

\begin{abstract}
Exact distributions of generalized Chi-square and Fisher statistics are used to derive confidence intervals and significance tests for inferring on one or two scaling parameters, respectively, under non-standard assumptions w.r.t. the multivariate sample distribution. The latter may have convex or radially concave density level sets and heavy or light distribution centers and tails. Independent and $I_{n, p}$-dependent sample variables are considered.

Keywords: Estimating and testing scaling parameters, Non-standard model assumptions, Generalized Chi-square and Fisher distributions, Heavy and light distribution tails, Heavy and light distribution centers, Independence sampling, Dependence sampling

MSC2010: Primary: 62F03, 62F25, 62C07 secondary: 6E15
\end{abstract}

\section{Introduction}

The well known Chi-square and Fisher distributions were introduced already a long time ago in (Fisher 1924a, b;1925; Hermert 1876; Pearson 1900), respectively. Since then, methods of statistical inference on variances are extensively studied under various aspects in numerous papers, see e.g. (Mirvaliev 1987; Plackett 1983; Statlect 2016) and the recent survey in (Bolboaca et al. 2011), for only to mention a few of them.

It is often natural to assume that expectations are unknown and thus to additionally be estimated when studying variances. In some cases, however, it may be reasonable to assume that expectations are known. Assume, for example, a company produces tubes of a certain norm length. The production dependent deviations from this length may according to (Bomsdorf 1989), p. 162 be considered as normally distributed with expectation zero and unknown variance. If the company indicates to customers a certain variance of tube length then one might be interested in proving this.

The successful fulfillment of manufacturing orders requires according to (Haidenhain 2011) machine tools with high thermal stability. Rapidly changing operating conditions for machine tools, however, may lead to unknown variances of technical features having a known expectation and make it difficult to constantly ensure accuracy in production processes.

Moreover, comparing in another situation instrumental accuracy by measuring a certain standard several times with two gages may generate random samples having the same

(c) 2016 Richter. Open Access This article is distributed under the terms of the Creative Commons Attribution 4.0 International License (http://creativecommons.org/licenses/by/4.0/), which permits unrestricted use, distribution, and reproduction in any medium, provided you give appropriate credit to the original author(s) and the source, provide a link to the Creative Commons license, and indicate if changes were made. 
known mean and unknown variances which one might be interested then to compare with each other.

If in such situations independent random variables $X_{1}, \ldots, X_{n}$ and $Y_{1}, \ldots, Y_{m}$ follow Gaussian laws with known expectations $\mu_{1}, \mu_{2}$ and unknown standard deviations $\sigma_{1}, \sigma_{2}$, respectively, then, with $\hat{\sigma}_{1}^{2}=\frac{1}{n} \sum_{1}^{n}\left(X_{i}-\mu_{1}\right)^{2}$ being the maximum likelihood estimator of the variance $\sigma_{1}^{2}, n\left(\frac{\hat{\sigma}_{1}}{\sigma_{1}}\right)^{2}$ follows a Chi-square density with $n$ d.f., and $\frac{\left(\hat{\sigma}_{1} / \sigma_{1}\right)^{2}}{\left(\hat{\sigma}_{2} / \sigma_{2}\right)^{2}}$ follows Fisher's $F$-distribution with $(n, m)$ d.f.

The aim of the present paper is to allow more general distributions of the sample vector, and to study exact distributions of generalized Chi-square and Fisher statistics being based upon the maximum likelihood estimators of the model's scaling parameters. The multivariate sample distributions considered to this end are convex or radially concave contoured. These two distribution classes are subclasses of the family of star-shaped distributions.

The paper is organized as follows. Star-shaped distributions are introduced in Section 2. A one sample non-standard scaling model for i.i.d. power exponentially distributed random variables and a certain extension of it are introduced in Section 3.1. Confidence estimation of and significance testing of hypotheses on the scaling parameter are discussed in Sections 3.2 and 3.4 for $p$-power exponential and more general sample distributions, respectively. Preparations for the latter case are given in Section 3.3. A two sample non-standard scaling parameter model ist studied then in Section 3.5 where estimation and testing are dealt with. A brief comparison of norms and antinorms, generating convex or radially concave level sets of the multivariate sample density, respectively, is given in the Appendix.

Finally, we remark that an introduction to non-standard models and exact distribution theory is given in (Richter 2012, 2014). For closely related studies on expectations and variances based upon $l_{n, p}$-norms and goodness of fitting using $l_{n, p}$-norms for data sets that follow a power exponential distribution, we refer to (Livadiotis 2007, 2012, 2014). For a comparison of the variances of two dependent random variables, see (Wilcox 2015).

\section{Star-shaped sample distributions}

\subsection{The general class}

The most general non-standard distributions of the multivariate sample vector we are considering in the present paper are star-shaped distributions. More specific distributions are $l_{n, p}$-symmetric and $p$-generalized elliptically contoured distributions considered, e.g., in (Richter 2012, 2014), respectively. Let $K \subset \mathbb{R}^{n}$ denote a star body with the origin being an inner point, and let $S$ be its topological boundary which will be called a star sphere. The Minkowski functional of $K$ is defined by $h_{K}(x)=\inf \{\lambda>0: x \in \lambda K\}, x \in \mathbb{R}^{n}$ and satisfies $h_{K}(x)=1, x \in S$. Assume that a random vector $X$ defined on a probability space $(\Omega, \mathfrak{A}, P)$ allows the stochastic representation

$$
X \stackrel{d}{=} R \cdot U_{S}
$$

where $R$ is a non-negative random variable and $U_{S}$ follows independently of $R$ the $S$ generalized uniform distribution $\omega_{S}$ on $S$. Then $X$ is called origin-including star-shaped distributed. Note that $X \stackrel{d}{=} Y$ means that $X$ and $Y$ follow the same distribution law, and

$$
\omega_{S}(A)=\frac{O_{S}(A)}{O_{S}(S)}, A \in \mathfrak{B}_{S}=\mathfrak{B}^{n} \cap S
$$


where $\mathfrak{B}^{n}$ denotes the Borel $\sigma$-field in $\mathbb{R}^{n}$ and $O_{S}$ the star generalized surface content measure on $\mathfrak{B}_{S}$. If $\mu$ stands for the Lebesgue measure on $\mathfrak{B}^{n}$ and $C P C(A)=\left\{x \in \mathbb{R}^{n}\right.$ : $\left.x / h_{K}(x) \in A\right\}$ is the central projection cone generated by $A \in \mathfrak{B}_{S}$ then the star generalized surface content of $A$ is formally defined for a particular class of star spheres $S$ in (Richter 2007) and under the weak technical Assumption 1 for general star spheres in (Richter 2014) by

$$
O_{S}(A)=f_{A}^{\prime}(1)
$$

where

$$
f_{A}^{\prime}(r)=\frac{d}{d r} f_{A}(r), \quad f_{A}(r)=\mu(\operatorname{sector}(A, r)), \quad \operatorname{sector}(A, r)=C P C(A) \cap K(r)
$$

and $K(r)=r \cdot K=\left\{\left(r x_{1}, \ldots, r x_{n}\right)^{T} \in \mathbb{R}^{n}:\left(x_{1}, \ldots, x_{n}\right)^{T} \in K\right\}$. Integral, or differential geometric, characterizations of $O_{S}$ which can equivalently be taken as a definition of $O_{S}$ are given for particular classes of star bodies in (Richter 2009, 2013) and for arbitrary two-dimensional convex star bodies in (Richter 2015b). Note that, e.g., the well known (Richter 2007) $l_{n, p}$-generalized (non-Euclidean) surface content of the $l_{n, p}$-unit sphere,

$$
\omega_{n, p}=\frac{2^{n} \Gamma\left(\frac{1}{p}\right)^{n}}{p^{n} \Gamma\left(\frac{n}{p}\right)}, p>0,
$$

is equal to the Euclidean surface content, if and only if $p=2$.

There are widespread miss-understandings w.r.t. this circumstance in the literature. The reader should be aware that there is some literature where formulas are derived which are called surface content and uniform distribution, respectively, however neither defining in advance the notion of surface content nor referring to literature where such definition is given.

The star generalized surface measure on the boundary of $n$-dimensional convex or radially concave star bodies is dealt with under different aspects in (Richter 2016, c, 2016). A general result in (Richter 2016) says that

$$
O_{S}(A)=\int_{G(A)} \hat{h}_{\hat{K}}(N(x)) d x
$$

where

$$
\hat{K}=\{\lambda x: x \in \operatorname{Grad}(S), 0 \leq \lambda \leq 1\}, \quad \operatorname{Grad}(S)=\left\{\nabla h_{K}(x), x \in S\right\}
$$

and

$$
\hat{h}_{\hat{K}}(t)=\inf \{\lambda>0: t \in \lambda \hat{K}\}, t \in \operatorname{pos} \hat{K}
$$

is a certain modification of the Minkowski functional of $\hat{K}$. Here, $\operatorname{pos} x=\{\lambda x: \lambda \geq 0\}, x \in$ $\mathbb{R}^{n}$ and $\operatorname{pos} M=\bigcup_{x \in M} \operatorname{pos} x$.

If $X$ is origin-including star-shaped distributed and $v \in \operatorname{st}(K)$ where $s t(K)$ denotes the set of all points w.r.t. which $K$ is star-shaped then $X-v$ is also origin-including starshaped distributed. If, however, $v$ does not belong to $s t(K)$ then $X-v$ will be called just star-shaped distributed. The first of these two cases is dealt with in (Richter 2014), Section 5.1 where non-concentric elliptically contoured distributions are introduced. Both cases are considered when studying non-central Chi-square distributions in (Ittrich et al. 2000). 
For a density having non-concentric circular contours with centers on a straight line, see (Arnold et al. 2008).

Example. Let $a=\left(a_{1}, \ldots, a_{n}\right)^{T} \in \mathbb{R}^{n}$ be a vector having positive components, $p \geq$ 1 , and $K=\left\{x \in \mathbb{R}^{n}:\left(\sum_{i=1}^{n}\left|\frac{x_{i}}{a_{i}}\right|^{p}\right)^{1 / p} \leq 1\right\}$. Then, according to (Richter 2014), the stargeneralized surface content measure on the boundary $S$ of $K$ is

$$
O_{S}(A)=a_{n} \int_{G(A)} \frac{d\left(x_{1}, \ldots, x_{n-1}\right)}{\left(1-\sum_{i=1}^{n}\left|\frac{x_{i}}{a_{i}}\right|^{p}\right)^{1-1 / p}}, A \in \mathfrak{B}_{S} .
$$

The special case $p=2$ has been dealt with in (Richter 2013).

\subsection{Norm contoured distributions}

Note that a symmetric convex star body having the origin as an inner point can always be generated by a norm, $h_{K}()=.\|$.$\| , and that according to (Richter 2015c)$

$$
O_{S}(A)=\int_{G(A)}\|N(x)\|^{*} d x, A \in \mathfrak{B}_{S}
$$

where $G(A)=\left\{\vartheta \in \mathbb{R}^{n-1}: \exists \eta=\eta(\vartheta)\right.$ s.t. $\left.\left(\vartheta^{T}, \eta(\vartheta)\right)^{T} \in A\right\}$ and $\|.\|^{*}$ denotes the norm dual to $\|$.$\| and N(\vartheta)=\left(\nabla \eta(\vartheta)^{T},-1\right)^{T}$ is the outer normal vector to $S$ at $\left(\vartheta^{T}, \eta(\vartheta)\right)^{T}$. If $K$ is the star body from the Example in Section 2.1 then (3) takes the specific form (2).

Let us emphasize that the star generalized surface content of an arbitrary measurable subset $A$ of the star sphere $S$ is a sum of integrals, in general. If $S^{+}$and $S^{-}$, however, denote upper and lower half spheres of the norm sphere $S$, and $K^{*}=\left\{y \in \mathbb{R}^{n}:\|y\|^{*} \leq 1\right\}$, then

$$
O_{S}(A)=\int_{G\left(A \cap S^{+}\right)} h_{K^{*}}(N(x)) d x+\int_{G\left(A \cap S^{-}\right)} h_{K^{*}}(N(x)) d x, A \in \mathfrak{B}_{S}
$$

where

$$
G\left(A \cap S^{+(-)}\right)=\left\{\vartheta \in \mathbb{R}^{n-1}: \exists \eta=\eta(\vartheta) \text { s.t. }\left(\vartheta^{T}, \eta\right)^{T} \in A \cap S^{+(-)}\right\} .
$$

For simplicity, and in accordance with Definition 1, Lemma 1 and Corollary 1 in (Richter 2014), however, $O_{S}(A)$ will be represented throughout this paper symbolically by a single integral.

Finally note that formula (1) applies whenever it is possible to evaluate the volume of a convex body. W.r.t. this research area we refer, e.g., to (Pisier 1989) and a discussion on page 11 in (Richter 2011).

Note that $K^{*}$ is always a symmetric convex star body and defines therefore a metric geometry in the sense of (Burago et al. 2001).

\subsection{Antinorm contoured distributions}

If $K$ is radially concave w.r.t. a fan $\mathcal{F}=\left\{C_{1}, C_{2}, \ldots\right\}$ and belongs to the particular class AN1 of star bodies introduced in (Richter 2015c) then its Minkowski functional is an 
antinorm, $h_{K}()=.\nmid . \nmid$. For the notion of antinorm, we refer to (Moszyńska and Richter 2012) and the Appendix. Let

$$
h_{K}^{\mathcal{F}}(u)=\sum_{i} I_{C_{i}}(u) \inf \left\{u^{T} y: y \in S \cap C_{i}\right\}, u \in \mathbb{R}^{n}
$$

be the anti-support function of $K$ w.r.t. $\mathcal{F}$ and

$$
K^{o}=\left\{\lambda(u) u: 0 \leq \lambda(u) h_{K}^{\mathcal{F}}(u) \leq 1, u \in S_{E}^{(n-1)}\right\}
$$

the anti-polar set of $K$ where $S_{E}^{(n-1)}$ denotes the Euclidean unit sphere in $\mathbb{R}^{n}$ then according to (Richter 2015c)

$$
O_{S}(A)=\int_{G(A)} h_{K^{o}}(N(x)) d x
$$

where $N(x)$ is the inner normal vector to $S$ at $x \in S$.

Example. Let the antinorm ball and sphere $K$ and $S$, respectively, be formally defined as in the preceding example but with $p \in(0,1]$, and let $a$ still be a vector with positive components. Then the $S$-generalized surface content measure $\mathfrak{O}_{S}$ satisfies representation (2). Notice, however, that we are evaluating star generalized surface contents of subsets of antinorm spheres, here, while star generalized surface contents of subsets of norm spheres are considered in the preceding example. Notice that $1-1 / p>0$ if $p>1$ and $1-1 / p<0$ if $0<p<1$.

\subsection{Continuous distributions}

Let a function $g:[0, \infty) \rightarrow[0, \infty)$ satisfy the assumptions $0<I(g)<\infty$ where $I(g)=\int_{0}^{\infty} r^{n-1} g(r) d r$. Such function is called a density generating function (dgf). A dgf can be used modeling light or heavy distribution centers and tails. Moreover, it allows even modeling complete level distributions. Well known examples are power- $t$ exponential dgf $g_{P E}(r)=\exp \left\{-\frac{r^{p}}{p}\right\}$, Kotz type dgf $g_{K}(r)=r^{M-1} e^{-\beta r^{\gamma}}, \beta, \gamma>0,2 M+n>2$ and Pearson-VII-type dgf $g_{P T 7}(r)=\left(1+\frac{r}{m}\right)^{-M}, M>n / 2, m>0$ where $I\left(g_{P E}\right)=$ $p^{n / p-1} \Gamma(n / p), I\left(g_{K}\right)=\frac{\Gamma((n+M-1) / \gamma)}{\gamma \beta^{(n+M-1) / \gamma}}$ and $I\left(g_{P T 7}\right)=m^{n} B(M-n, n)$, respectively. Studentand Cauchy-type dgf's are special cases of $g_{P T 7}$.

If the star-shaped distributed random vector $X$ has a probability density (p.d.), $\varphi_{g, K, \nu}$ say, then

$$
\varphi_{g, K, v}(x)=C(g, K) g\left(h_{K}(x-v)\right), x \in \mathbb{R}^{n} .
$$

Densities of this type have been considered in (Arnold et al. 2008; Fernández et al. 1995). Here,

$$
C(g, K)=1 /\left(I(g) O_{S}(S)\right)
$$

and the p.d. of the generating variate $R$ is

$$
f(r)=I_{(0, \infty)}(r) r^{n-1} g(r) .
$$


The corresponding probability distribution allows the geometric measure representation

$$
\Phi_{g, K, \nu}(B)=\frac{1}{I(g)} \int_{0}^{\infty} r^{n-1} g(r) \mathfrak{F}_{S}(B, r) d r, B \in \mathfrak{B}^{n}
$$

where

$$
\mathfrak{F}_{S}(B, r)=\omega_{S}\left(\left[\frac{1}{r}(B-v)\right] \cap S\right), r>0
$$

denotes the intersection proportion function (ipf) of the set $B$.

If $K$ is the Euclidean unit ball then $K^{*}=K$ and $O_{S}$ is the Euclidean surface content measure. In this case, representation (6) has been considered already in (Richter 1985, 1991). For specific non-Euclidean cases, (6) has been considered in (Richter 2007, 2009, 2013, 2014).

Surveys of different types of applications of formula (6) are given for $n=2$ in (Richter 2015b) and for arbitrary $n$ in (Richter 2012, 2014). For two elementary applications, see Section 2.6.

\subsection{Building subclasses and class extensions}

\subsubsection{Building subclasses of distributions by modeling $R$}

Let us denote the probability law of the generating variate $R$ by $P^{R}$, and let $v$ be a $\sigma$-finite measure on the Borel $\sigma$-field $\mathfrak{B}^{(1)}$ (or its restriction to the positive real line) such that $P^{R}$ is absolutely continuous w.r.t. $v$. If $v$ is the Lebesgue measure on $\mathfrak{B}^{(1)}$ then $\frac{d P^{R}}{d v}(r)=f(r)$ is the p.d. considered in Section 2.4. In Sections 2.2 and 2.3, $P^{R}$ can be chosen as the distribution of any non-negative random variable thus $\frac{d P^{R}}{d v}$ being a mixture of an absolutely continuous, a discrete and a singular component, in general.

\subsubsection{Extending distribution classes by restricting $U$}

We emphasize for the rest of this section that $S$ circumscribes the origin as in inner point. Let $\mathcal{M}$ be a measurable subset of the star sphere $S$. We call a random vector $U_{\mathcal{M}}$ stargeneralized uniformly distributed on $\mathcal{M}$ (or the $\mathcal{M}$-restriction of $U$ ) if $U_{\mathcal{M}}$ follows the distribution law

$$
P^{U_{\mathcal{M}}}(A)=\frac{O_{S}(A)}{O_{S}(\mathcal{M})}, A \in \mathcal{B}_{S} \cap \mathcal{M}
$$

Note that $U_{S} \stackrel{d}{=} U$ and that $\operatorname{pos} \mathcal{M}$ is not an absorbing set unless for $\mathcal{M}=S$.

For an example of an $\mathcal{M}$-restriction of $U$ occuring in depth-based classification for distributions with non-convex support, we refer to (Hlubinka and Vencalek 2013).

We call

$$
\mathcal{D}_{S}(\mathcal{M})=\left\{\mathcal{L}\left\{R \cdot U_{\mathcal{M}}\right\}, R \text { is a non-negative random variable }\right\}
$$

where $\mathfrak{L}(Y)$ means the distribution law of $Y$ the class of directionally $\mathcal{M}$-out-of-S restricted distributions and

$$
\mathcal{D}_{S}=\left\{\mathcal{D}_{S}(\mathcal{M}): \mathcal{M} \text { is a measurable subset of the star sphere } S\right\}
$$

the class of directionally out-of- $S$ restricted distributions. Finally,

$$
\mathcal{D}=\left\{\mathcal{D}_{S}: S \text { is a star sphere }\right\}
$$

is called the class of directionally out-of a star sphere restricted distributions in $\mathbb{R}^{n}$. 


\subsection{Two elementary types of applications of formula (6)}

\subsubsection{The ipf is the indicator function of an interval}

Assume that the random vector $X$ follows the continuous star-shaped distribution law $\Phi_{g, K, v}$, and let the statistic $T: \mathbb{R}^{n} \rightarrow[0, \infty)$ be defined by $T(x)=h_{K}(x-v)$. The ipf of the set $B(t)=\left\{x \in \mathbb{R}^{n}: T(x)<t\right\}$ allows then the representation

$$
\mathfrak{F}_{S}(B(t), r)=\frac{O_{S}\left(\left[\frac{1}{r}(B(t)-v)\right] \cap S\right)}{O_{S}(S)}= \begin{cases}1 & r \in I(t) \\ 0 & \text { elsewhere }\end{cases}
$$

where $S$ is still the boundary of $K$ and $I(t)$ is the particular interval [0,t). It follows from (6) that

$$
P(T(X)<t)=\Phi_{g, K, v}\left(\left\{x \in \mathbb{R}^{n}: h_{K}(x-v)<t\right\}\right)=\frac{1}{I(g) O_{S}(S)} \int_{0}^{\infty} r^{n-1} g(r) I_{[0, t)} d r .
$$

Now, a density formula can be derived from the representation

$$
P(T(X)<t)=\frac{1}{I(g) O_{S}(S)} \int_{I(t)} r^{n-1} g(r) d r .
$$

Similar results hold for arbitrary intervals $I(t)$. For a specific application of the present type we refer to the calculation of generalized Chi-square densities.

\subsubsection{The ipf is constant}

Let $\mathfrak{F}_{B(t)}(r)=C(t)$ where $C(t) \in(0,1)$ does not depend on $r$. Then it follows from (6) that $P(T<t)=C(t)$, and $T$ is called a $g$-robust statistic. For specific such applications we refer to the geometric derivation of generalized Student and Fisher distributions. The notion of robustness considered here was studied already in (Fang et al. 1990) for elliptically contoured distributions.

Several other types of sets for which the ipf has been calculated already in the past can be found in some of author's papers surveyed in (Richter 2012, 2014).

\section{Inference on scaling parameters}

\subsection{The power exponential sample distribution}

\subsubsection{A basic scaling parameter model}

Let the i.i.d. random variables $X_{1}, \ldots, X_{n}$ follow the power exponential (or $p$-generalized Gaussian or $p$-generalized Laplace) density $f_{p}(. ; \mu, \sigma)$, where $p>0$ and $\mathbb{E} X_{1}=\mu$ are known, and

$$
f_{p}(x ; \mu, \sigma)=\frac{C_{p}}{\sigma} \exp \left\{-\frac{|x-\mu|^{p}}{p \sigma^{p}}\right\},-\infty<x<\infty .
$$

Note that $C_{p}=p^{1-1 / p} /(2 \Gamma(1 / p))$ and $V\left(X_{1}\right)=\sigma^{2} \Gamma\left(\frac{3}{p}\right) / \Gamma\left(\frac{1}{p}\right)$. Let $X_{0}$ follow the p.d. of the standardized $p$-generalized normal distribution, $f_{p}(. ; 0,1)$. Then $X_{1}$ satisfies the representation $X_{1} \stackrel{d}{=} \mu+\sigma X_{0}$. The quantity $\sigma$ is called a scaling parameter.

\subsubsection{Norm and antinorm contoured sample distributions}

The density of the sample vector $X=\left(X_{1}, \ldots, X_{n}\right)$ is

$$
\prod_{i=1}^{n} f_{p}\left(x_{i} ; \mu, \sigma\right)=\frac{C_{p}^{n}}{\sigma^{n}} \exp \left\{-\sum_{i=1}^{n} \frac{\left|x_{i}-\mu\right|^{p}}{p \sigma^{p}}\right\}, x=\left(x_{1}, \ldots, x_{n}\right) \in \mathbb{R}^{n} \text {. }
$$


The density super level sets $\left\{x \in \mathbb{R}^{n}: \prod_{i=1}^{n} f_{p}\left(x_{i} ; \mu, \sigma\right) \geq t\right\}, t>0$ are generated by the functional

$$
x \rightarrow|x|_{p}=\left(\sum_{i=1}^{n}\left|x_{i}\right|^{p}\right)^{1 / p}
$$

which is a norm if $p \geq 1$ and an antinorm if $0<p \leq 1$. Here, $K=B_{p}=\left\{x \in \mathbb{R}^{n}:|x|_{p}\right.$ $\leq 1\}$.

For getting a more concrete idea of the shape of this density let us recall that the boundaries of the two-dimensional density super level sets are the star circles

$$
\left\{\left(x_{1}, x_{2}\right)^{T} \in \mathbb{R}^{2}:\left|x_{1}\right|^{p}+\left|x_{2}\right|^{p}=r^{p}\right\}, r>0 .
$$

The star discs circumscribed by these $p$-circles are convex if $p \geq 1$ and radially concave if $0<p \leq 1$. For the latter notion, we refer to (Moszyńska and Richter 2012). Similar properties hold in the case of arbitrary finite dimension.

In the geometric measure representation (6), the star generalized surface content measure allows for every $p>0$ and every measurable subset $A$ of the upper (or lower) half of the star sphere $S$ the representation

$$
O_{S}(A)=\int_{G(A)}\left(1-\sum_{1}^{n-1}\left|x_{i}\right|^{p}\right)^{(1-p) / p} d x
$$

for $p \geq 1$ and $p \in(0,1]$ being each time a special case of (3) and (4), respectively.

\subsubsection{Extended scaling parameter model}

Let now $X_{1}, \ldots, X_{n}$ be i.i.d. random variables following the densities

$$
f_{p}\left(x ; \mu_{i}, \sigma^{2} a_{i}^{2}\right)=\frac{C_{p}}{\sigma a_{i}} \exp \left\{-\frac{\left|x-\mu_{i}\right|^{p}}{p\left(\sigma a_{i}\right)^{p}}\right\}, x \in \mathbb{R}, i=1, \ldots, n
$$

where $\mathbb{E} X_{i}=\mu_{i} \in \mathbb{R}, p>0, a_{i}>0$ are known, and $\sigma>0$ is unknown. Note that

$$
\mathbb{V}\left(X_{i}\right)=\sigma^{2} a_{i}^{2} \frac{\Gamma(3 / p)}{\Gamma(1 / p)}
$$

and that $X_{i}$ allows the representation

$$
X_{i} \stackrel{d}{=} \mu_{i}+\sigma a_{i} X_{0}
$$

where $X_{0}$ has the p.d. $f_{p}(. ; 0,1)$ and $\sigma$ is a common scaling parameter.

\subsection{Inference on a single scaling parameter}

\subsubsection{Basic scaling parameter model.}

The maximum likelihood estimator of $\sigma$ in the model (7) is the sufficient statistic

$$
\hat{\sigma}=\operatorname{mle}(\sigma)=\left(\frac{1}{n} \sum_{i=1}^{n}\left|X_{i}-\mu\right|^{p}\right)^{1 / p} .
$$

Note that

$$
\mathbb{E}\left(\hat{\sigma}^{p}\right)=\sigma^{p} \text { and } \mathbb{V}\left(\hat{\sigma}^{p}\right)=\frac{p}{n} \sigma^{2 p},
$$

$\hat{\sigma}^{p}$ thus being an unbiased and consistent estimator for $\sigma^{p}$. The distribution of $\hat{\sigma}$ can be derived, e.g., on using formula (6) with the help of formula (8). The result follows below from the consideration for the extended scaling parameter model (9). 
In the sense of Section 2.6, it is an elementary problem to evaluate the $S$-generalized surface content of subsets of $S$ according to (8) in the present case because we are dealing here with a set the ipf of which is just the indicator function of an interval. This way it is proved (within a more general framework) in (Richter 2009) (and with a slight modification already in (Richter 2007)) that $n(\hat{\sigma} / \sigma)^{p}$ follows the $\chi^{p}$-(or $p$-generalized $\chi^{2}-$ ) distribution with $n$ d.f., $n(\hat{\sigma} / \sigma)^{p} \sim \chi^{p}(n)$ where $Y \sim Q$ means that the random variable $Y$ follows the probability distribution $Q$. The p.d. of the $\chi^{p}(n)$ distribution is according to (Richter 2007, 2009)

$$
f_{n, p}(t)=\frac{t^{n / p-1} e^{-t / p}}{p^{n / p} \Gamma(n / p)} I_{(0, \infty)}(t) .
$$

Although assigning in its derivation the number $\omega_{n, p}$ the Euclidean interpretation instead of the right non-Euclidean one, density (10) was derived later again in (Livadiotis 2012). For a considerable generalization of this density, see (14) below and (Richter 2009). We denote the quantile of order $q \in(0,1)$ of the $\chi^{p}(n)$ distribution by $\chi_{q}^{p}(n)$. Thus, by definition $P\left(n(\hat{\sigma} / \sigma)^{p}<\chi_{q}^{p}(n)\right)=q$. Note that the $\chi^{p}(n)$ distribution is a particular Gamma distribution having parameters $\frac{n}{p}$ and $\frac{1}{p}$. The quantiles of this distribution can therefore be computed using the inverse Gamma function being available in standard software.

\subsubsection{Confidence intervals for $\sigma^{p}$}

The random intervals

$$
\left[\frac{\left|X-\mu 1_{n}\right|_{p}^{p}}{\chi_{1-\alpha_{1}}^{p}(n)}, \frac{\left|X-\mu 1_{n}\right|_{p}^{p}}{\chi_{\alpha_{2}}^{p}(n)}\right],\left[\frac{\left|X-\mu 1_{n}\right|_{p}^{p}}{\chi_{1-\alpha}^{p}(n)}, \infty\right) \text { and }\left(-\infty, \frac{\left|X-\mu 1_{n}\right|_{p}^{p}}{\chi_{\alpha}^{p}(n)}\right]
$$

are level $1-\alpha$ two-sided, and one-sided upper and lower confidence intervals for $\sigma^{p}$, respectively, with $\alpha=\alpha_{1}+\alpha_{2}$ in the two-sided case, and where $1_{n}=(1, \ldots, 1)^{T} \in \mathbb{R}^{n}$. If $p=2$ then $\chi_{q}^{p}(n)$ is the common quantile of order $q$ of the Chi-square distribution with $n$ d.f., $\chi_{q}^{2}(n)$. The case $p=1$ is dealt with in (Kotz et al. 2001), formula (2.6.112), where, however, $2 / \chi_{1-\alpha / 2}^{2}(2 n)$ is used instead of $1 / \chi_{1-\alpha / 2}^{1}(n)$. Tables $2,3,4$ and 5 present quantiles $\chi_{q}^{p}(n)$ for several values of $n, p$ and $q$.

\subsubsection{Significance testing}

If one wants to test the hypothesis $H_{0}: \sigma=\sigma_{0}$ versus the two-sided alternative $H_{A}: \sigma \neq$ $\sigma_{0}$ then the decision rule of a level- $\alpha$ significance test is defined as to reject $H_{0}$ if

$$
\sum_{i=1}^{n}\left|\frac{X_{i}-\mu}{\sigma_{0}}\right|^{p} \in\left(-\infty, \chi_{\alpha_{2}}^{p}(n)\right) \bigcup\left(\chi_{1-\alpha_{1}}^{p}(n), \infty\right),
$$

where $\alpha=\alpha_{1}+\alpha_{2}, 0<\alpha_{i}<1 / 2, i=1,2$. Tests for one-sided hypotheses are analogously constructed.

\subsubsection{Extended scaling parameter model}

In the model (9), the maximum likelihood estimator of the common scaling parameter $\sigma$ is

$$
\operatorname{mle}(\sigma)=\widehat{\sigma}=\frac{1}{n^{1 / p}}|X-v|_{a, p}
$$

where $v=\left(\mu_{1}, \ldots, \mu_{n}\right)^{T}$ and

$$
|x|_{a, p}=\left(\sum_{i=1}^{n}\left|\frac{x_{i}}{a_{i}}\right|^{p}\right)^{1 / p} x \in \mathbb{R}^{n} .
$$


The p.d. of $X$ is called a $p$-generalized axes aligned elliptically contoured density having the contour defining star body $K=B_{a, p}=\left\{x \in \mathbb{R}^{n}:|x|_{a, p} \leq 1\right\}$, see (Richter 2014). This density, as well as $\hat{\sigma}$ allow functional representations

$$
f_{X}(x)=\frac{C_{p}^{n}}{\sigma^{n} a_{1} \cdot \ldots \cdot a_{n}} g_{P E}\left(h_{\sigma B_{a, p}}(x-v)\right), x \in \mathbb{R}^{n}
$$

and

$$
\widehat{\sigma}=\frac{1}{n^{1 / p}} h_{B_{a, p}}(X-v) .
$$

For the derivation of the density of $n\left(\frac{\hat{\sigma}}{\sigma}\right)^{p}$, we start from the equation

$$
P\left(n\left(\frac{\widehat{\sigma}}{\sigma}\right)^{p}<t\right)=\Phi_{g_{P E}, B_{a, p}, 0_{n}}\left(t^{1 / p} B_{a, p}\right) .
$$

We recall that the intersections $B \cap S_{a, p}(r), r>0$, where $S_{a, p}(r)=r S_{a, p}$ and $S=S_{a, p}$ is the boundary of $K=B_{a, p}$ are called sometimes the 'indivisibles' of a set $B$.

The geometric disintegration method behind formula (6) divides integration into first an integration on a part of a generalized sphere and second an integration w.r.t. $r$ :

$$
P\left(n\left(\frac{\widehat{\sigma}}{\sigma}\right)^{p}<t\right)=\frac{1}{I\left(g_{P E}\right)} \int_{0}^{\infty} r^{n-1} g_{P E}(r) \mathfrak{F}_{S_{a, p}}\left(t^{1 / p} B_{a, p}, r\right) d r
$$

where according to Section 2.6

$$
\mathfrak{F}_{S_{a, p}}\left(t^{1 / p} B_{a, p}, r\right)=I_{\left[0, t^{1 / p}\right]}(r) .
$$

Thus

$$
P\left(n\left(\frac{\widehat{\sigma}}{\sigma}\right)^{p}<t\right)=\frac{1}{I\left(g_{P E}\right)} \int_{0}^{t^{1 / p}} r^{n-1} g_{P E}(r) d r, t>0 .
$$

Note that this result does not depend on $a=\left(a_{1}, \ldots, a_{n}\right)^{T}$. Finally,

$$
n\left(\frac{\widehat{\sigma}}{\sigma}\right)^{p} \sim \chi^{p}(n) .
$$

If one replaces the functional $|\cdot|_{p}^{p}$ by $|\cdot|_{a, p}^{p}$ in the definitions of the intervals (11) then the new intervals are confidence intervals for $\sigma^{p}$ in the extended scaling parameter model (9).

\subsection{The $p$-generalized Student's sample distribution and beyond}

We study here the distribution of the statistic $n\left(\frac{\hat{\sigma}}{\sigma}\right)^{p}$ if the distribution of the sample vector $X=\left(X_{1}, \ldots, X_{n}\right)^{T}$ is not the $n$-dimensional power exponential one but any other element of the family of $l_{n, p}$-symmetric distributions. Before doing this, let us recall that one of the most famous non-normal spherically contoured distributions is the Student's $t$-distribution because of its heavy tails and the remarkable property that all its marginal distributions have a density generating function of the same known type. Moreover, note that the components of a spherically contoured vector are uncorrelated but not independent unless in the Gaussian case. All these properties hold also true for the $p$-generalized Student's $t$-distribution with $f$ degrees of freedom which was studied for dimension one in (Richter 2007) and for arbitrary finite dimension $n$ in Arellano-Valle and Richter (2012). 
In the latter case, the dgf may be chosen as

$$
g_{S t(n, p, f)}(r)=\left(1+\frac{r^{p}}{f}\right)^{-\frac{f+n}{p}}, r>0,
$$

and the density of $X$ is

$$
t_{n, p}(x ; f)=D_{n, p, f}\left(1+\frac{|x|_{p}^{p}}{f}\right)^{-\frac{f+n}{p}}, x \in \mathbb{R}^{n}
$$

where $D_{n, p, f}=\frac{\left(\frac{p}{2}\right)^{n} \Gamma\left(\frac{n+f}{p}\right)}{\Gamma\left(\frac{f}{p}\right)\left(\Gamma\left(\frac{1}{p}\right)\right)^{n} f^{n / p}}$. It follows that the density of the statistic $n\left(\frac{\hat{\sigma}}{\sigma}\right)^{p}$ is then

$$
f_{n, g_{S t}, p}^{\chi}(t)=\frac{t^{\frac{n}{p}-1}}{f^{\frac{n}{p}} B\left(\frac{n}{p}, \frac{f}{p}\right)\left(1+\frac{t^{p}}{f}\right)^{\frac{f+n}{p}}}, t \in \mathbb{R} .
$$

If, however, the density of the sample vector $X$ is an arbitrary $l_{n, p^{-}}$symmetric one having $\operatorname{dgf} g$,

$$
f(x)=C(g, p) g\left(|x|_{p}\right), x \in \mathbb{R}^{n},
$$

then, according to Richter (2009),

$$
f_{n, g, p}^{\chi}(t)=\frac{t^{\frac{n}{p}-1} g(t)}{p I_{n, g, p}}, t \in \mathbb{R},
$$

where $I_{n, g, p}=\int_{0}^{\infty} r^{n-1} g\left(r^{p}\right) d r$.

This density can be considered as a $(p, g)$-generalization of the classical Chi-square density with $n$ d.f., or as a $g$-generalization of the $p$-generalized Chi-square density $f_{n, p}$ in (10). We denote the quantile of order $q$ of the $\chi_{g}^{p}(n)$-distribution by $\chi_{g ; q}^{p}(n), 0<q<1$, that is $\chi_{g}^{p}(n)\left(\chi_{g ; q}^{p}(n)\right)=q$. Note that $\chi_{g_{P E} ; q}^{p}(n)=\chi_{q}^{p}(n)$.

Let a random variable following density (14) be denoted by $R^{p}$. Note that tables of quantiles of the distribution of $R$ are recently provided in Müller and Richter (2016) for $n \in\{1,2,3\}, p \in\left\{\frac{1}{2}, 1,3\right\}$ and different dgfs $g$ of power-exponential and Student's type as well of Pearson type 2 .

\subsection{Inference for generalized sample distributions}

If the sample vector $X$ is distributed according to an $l_{n, p}$-symmetric distribution, see e.g. (Richter 2009), with a dgf $g$ being different from the power exponential one, $g \neq g_{P E}$, then $X_{1}, \ldots, X_{n}$ are not further independent. In this case of $l_{n, p}$-dependent observation variables, it is possible to study decision rules for exact inference on scaling parameters as before. Confidence and significance levels of the analogously defined estimators and tests, however, change in dependence of the actual shape of $g$. It is an immediate consequence from the equation

$$
P\left(\chi_{g, \alpha_{2}}^{p}(n)<n\left(\frac{\hat{\sigma}}{\sigma}\right)^{p}<\chi_{g, 1-\alpha_{1}}^{p}(n)\right)=1-\alpha_{1}-\alpha_{2}
$$

that the level- $\alpha$ confidence intervals in (11) extend to the present $g$-generalized case if all quantiles $\chi_{q}^{p}(n)$ are replaced with $\chi_{g ; q}^{p}(n)$. Similarly, the same replacement of quantiles $g$-generalizes the level- $\alpha$ tests considered in (12). 


\subsection{The ratio of two scaling parameters}

\subsubsection{The p-generalized Fisher statistic and Fisher distribution}

Let $X_{1}, \ldots, X_{n}$ and $Y_{1}, \ldots, Y_{m}$ be completely independent random variables with

$X_{i}$ having p.d. $f_{p}\left(. ; \mu_{1}, \sigma_{1}\right), i=1, \ldots, n$ and $Y_{i}$ having p.d. $f_{p}\left(. ; \mu_{2}, \sigma_{2}\right), i=1, \ldots, m$ where $p>0$ and the expectations $\mu_{1}, \mu_{2}$ are known and the scaling parameters $\sigma_{1}, \sigma_{2}$ are unknown. With the notation $X=\left(X_{1}, \ldots, X_{n}\right)^{T}, Y=\left(Y_{1}, \ldots, Y_{m}\right)^{T}$, the $n+m$ dimensional random vector $\left(X^{T} Y^{T}\right)^{T}$ follows the distribution $\Phi_{g, K, v}$ where the dgf is the power exponential one, $g(r)=g_{P E}(r)=I_{(0, \infty)}(r) \exp \left\{-\frac{r^{p}}{p}\right\}$, the star body $K$ is the $p$-generalized ellipsoid

$$
K=B_{p, \sigma_{1}, \sigma_{2}}=\left\{\left(x^{T} y^{T}\right)^{T} \in \mathbb{R}^{n+m}:\left|\left(x^{T} y^{T}\right)^{T}\right|_{p, \sigma_{1}, \sigma_{2}} \leq 1\right\},
$$

and the shift vector $v$ is $\left(\mu_{1} 1_{n}^{T} \mu_{2} 1_{m}^{T}\right)^{T} \in \mathbb{R}^{n+m}$. Here,

$$
\left|\left(x^{T} y^{T}\right)^{T}\right|_{p, \sigma_{1}, \sigma_{2}}=\left(\frac{|x|_{p}^{p}}{\sigma_{1}^{p}}+\frac{|y|_{p}^{p}}{\sigma_{2}^{p}}\right)^{1 / p},\left(x^{T} y^{T}\right)^{T} \in \mathbb{R}^{n+m} .
$$

Let, in accordance with Section 3.2,

$$
\hat{\sigma}_{1}^{p}=\frac{1}{n}\left|X-\mu_{1} 1_{n}\right|_{p}^{p}, \quad \hat{\sigma}_{2}^{p}=\frac{1}{m}\left|Y-\mu_{2} 1_{m}\right|_{p}^{p},
$$

and put

$$
T=\frac{\left(\hat{\sigma}_{1} / \sigma_{1}\right)^{p}}{\left(\hat{\sigma}_{2} / \sigma_{2}\right)^{p}}
$$

Note that, for $t>0$,

$$
\begin{aligned}
P(T<t) & =\Phi_{g_{P E}, B_{p ; \sigma_{1}, \sigma_{2}, v}}\left(\left\{\left(x^{T} y^{T}\right)^{T} \in \mathbb{R}^{n+m}: \frac{\left|\frac{x-\mu_{1} 1_{n}}{\sigma_{1}}\right|_{p}^{p}}{\left|\frac{y-\mu_{2} 1_{m}}{\sigma_{2}}\right|_{p}^{p}}<\frac{n}{m} t\right\}\right) \\
& =\Phi_{g_{P E}, B_{p ; 1,1}, 0}\left(C_{n, m ; p}(t)\right)
\end{aligned}
$$

where

$$
C_{n, m ; p}(t)=\left\{\left(x^{T} y^{T}\right)^{T} \in \mathbb{R}^{n+m}: \frac{|x|_{p}^{p}}{|y|_{p}^{p}}<\frac{n}{m} t\right\}
$$

denotes a cone in $\mathbb{R}^{n+m}$. The ipf of this cone is studied in Richter (2009). It is shown there that $C_{n, m ; p}(t)$ belongs to the second elementary type of sets considered in Section 2.6, thus

$$
P(T<t)=\mathfrak{F}_{S_{a, \sigma_{1}, \sigma_{2}}}\left(C_{n, m ; p}(t), 1\right), t>0
$$

does not depend on the $\operatorname{dgf} g$. To be specific,

$$
\mathfrak{F}_{S_{a, \sigma_{1}, \sigma_{2}}}\left(C_{n, m ; p}(t), 1\right)=\frac{p}{B\left(\frac{n}{p}, \frac{m}{p}\right)} \int_{\operatorname{arccot}\left(\left(\frac{n}{m} t\right)^{1 / p}\right)}^{\pi / 2} \frac{(\cos \varphi)^{n-1}(\sin \varphi)^{m-1}}{\left((\cos \varphi)^{p}+(\sin \varphi)^{p}\right)^{n / p}} d \varphi .
$$

Hence, the p.d. of $T$ is

$$
f_{n, m ; p}(t)=\frac{(n / m)^{n / p} t^{n / p-1}}{B(n / p, m / p)(1+n t / m)^{(n+m) / p}} I_{(0, \infty)}(t)
$$


The distribution law of $T$, denoted by $F_{n, m}(p)$, is called in Richter (2009) the $p$-generalized Fisher distribution with $(n, m)$ d.f. Finally, it turns out that

$$
T=\frac{m \sigma_{2}^{p}\left|X-\mu_{1} 1_{n}\right|_{p}^{p}}{n \sigma_{1}^{p}\left|Y-\mu_{2} 1_{m}\right|_{p}^{p}} \sim F_{n, m}(p) .
$$

Let us denote the $q$ 'th order quantile of the $p$-generalized Fisher distribution having $(n, m)$ d.f. by $F_{n, m ; q}(p), q \in(0,1)$

\subsubsection{Confidence intervals}

Two-sided, possibly asymmetric, and one-sided upper and lower confidence intervals for $\frac{\sigma_{1}^{p}}{\sigma_{2}^{p}}$ are, correspondingly,

$$
\xi\left[\frac{1}{F_{n, m ; 1-\alpha_{1}}(p)}, \frac{1}{F_{n, m ; \alpha_{2}}(p)}\right], \xi\left[\frac{1}{F_{n, m ; 1-\alpha}(p)}, \infty\right) \text { and } \xi\left(-\infty, \frac{1}{F_{n, m ; \alpha}(p)}\right]
$$

where $\xi[e, f]=\{\xi \cdot s: e \leq s \leq f\}$ and

$$
\xi=\frac{m\left|X-\mu_{1} 1_{n}\right|_{p}^{p}}{n\left|Y-\mu_{2} 1_{m}\right|_{p}^{p}}
$$

\subsubsection{Significance test}

For deciding

$$
H_{0}: \sigma_{1}=\sigma_{2} \text { vs. } H_{A}: \sigma_{1} \neq \sigma_{2}
$$

we use the distribution of $T$ if $H_{0}$ is true to construct a level $\alpha$ significance test. In this case, $T=\frac{m}{n} \frac{\left|X-\mu_{1} 1_{n}\right|_{p}^{p}}{\left|Y-\mu_{2} 1_{m}\right|_{p}^{p}}$. Reject $H_{0}$ if $T<F_{n, m ; \alpha_{2}}(p)$ or if $T>F_{n, m ; 1-\alpha_{1}}(p)$, respectively, where $\alpha_{1}>0, \alpha_{2}>0, \alpha_{1}+\alpha_{2}=\alpha<1$.

Proposition 1. If sample sizes $n$ and $m$ are equal then this rule is equivalent to rejecting $H_{0}$ if the likelihood ratio statistic is smaller than a constant $c \in(0,1)$ chosen s.t.

$P\left(\right.$ reject $H_{0}$ although $H_{0}$ is true $)=\alpha$.

To show this, let us consider the likelihood function

$$
L\left(\sigma_{1}, \sigma_{2}\right)=(n+m) \ln C_{p}-n \ln \sigma_{1}-m \ln \sigma_{2}-\frac{1}{p \sigma_{1}^{p}} \sum_{i=1}^{n}\left|x_{i}-\mu_{1}\right|^{p}-\frac{1}{p \sigma_{2}^{p}} \sum_{i=1}^{m}\left|y_{i}-\mu_{2}\right|^{p} .
$$

The maximum likelihood estimators of the scaling parameters are given in (15). If $\sigma_{1}=$ $\sigma_{2}=\sigma$, say, then $L^{*}(\sigma)=L(\sigma, \sigma)$ is the likelihood function for estimating $\sigma$, and

$$
\hat{\sigma}=\operatorname{mle}(\sigma)=\left(\frac{\sum_{i=1}^{n}\left|x_{i}-\mu_{1}\right|^{p}+\sum_{i=1}^{m}\left|y_{i}-\mu_{2}\right|^{p}}{n+m}\right)^{1 / p} .
$$

The likelihood ratio is

$$
\begin{aligned}
Q & =\frac{\sup _{\sigma_{1}=\sigma_{2}} L\left(\sigma_{1}, \sigma_{2}\right)}{\sup _{\sigma_{1}>0, \sigma_{2}>0} L\left(\sigma_{1}, \sigma_{2}\right)}=\frac{L^{*}(\hat{\sigma})}{L\left(\hat{\sigma}_{1}, \hat{\sigma}_{2}\right)} \\
& =\left(\frac{(n+m)^{n+m}}{n^{n} m^{m}}\right)^{1 / p} \frac{\left|x-\mu_{1} 1_{n}\right|_{p}{ }^{n}\left|y-\mu_{2} 1_{m}\right|_{p}^{m}}{\left(\left|x-\mu_{1} 1_{n}\right|_{p}{ }^{p}+\left|y-\mu_{2} 1_{m}\right|_{p}\right)^{(n+m) / p}} .
\end{aligned}
$$


Let $\xi=\left|x-\mu_{1} 1_{n}\right|_{p}^{p / 2}$ and $\eta=\left|y-\mu_{2} 1_{m}\right|_{p}^{p / 2}$. Because $n=m$ it follows that $Q \leq$ $t, t \in(0,1)$ iff $l(\xi) \geq 0$ where $l(\xi)=\xi^{2}+p \xi+q, p=-2 \eta / t^{p /(2 m)}$ and $q=\eta^{2}$. The two positive solutions of the equation $l(\xi)=0$ are

$$
\xi_{1}=-p / 2+\left(p^{2} / 4-q\right)^{1 / 2}=\frac{\eta}{t^{p /(2 m)}}\left(1+\left(1-t^{p / m}\right)^{1 / 2}\right),
$$

and

$$
\xi_{2}=-p / 2-\left(p^{2} / 4-q\right)^{1 / 2}=\frac{\eta}{t^{p /(2 m)}}\left(1-\left(1-t^{p / m}\right)^{1 / 2}\right)
$$

where $\eta>0$. Because $l\left(\right.$.) is a convex function, the rejection area of $H_{o}$ is the set of all $\xi$ satisfying $\xi \geq \xi_{1}$ or $\xi \leq \xi_{2}$. The latter inequalities are equivalent to

$$
\frac{\left|x-\mu_{1} 1_{n}\right|_{p}^{p}}{\left|y-\mu_{2} 1_{m}\right|_{p}^{p}} \geq \frac{2\left(1+\left(1-t^{p / m}\right)^{1 / 2}\right)}{t^{p / m}}-1
$$

and

$$
\frac{\left|x-\mu_{1} 1_{n}\right|_{p}^{p}}{\left|y-\mu_{2} 1_{m}\right|_{p}^{p}} \leq \frac{2\left(1-\left(1-t^{p / m}\right)^{1 / 2}\right)}{t^{p / m}}-1
$$

This proves the equivalence of the considered decision rules.

Remark 1. As we have seen in this section, inferring on the scaling parameters ratio in $l_{n, p}$-symmetric sample distributions can be done in a g-robust way. For a discussion of this property see Richter (2009), and note that it goes back in the case $p=2$ to Fang et al. (1990).

\section{Conclusions}

As to shortly summarize the results of this paper, we derived exact confidence intervals and significance tests for single scaling parameters and ratios of two such parameters if the $n$-dimensional sample vector follows a norm or antinorm contoured $l_{n, p}$-symmetric distribution. This assumption includes modeling heavy and light tails of the sample vector's distribution as well as independence and $l_{n, p}$-dependence sampling. Certain classical results are $(g, p)$-generalized in the present work. The additional methodological benefit coming from the present paper may be estimated having a look at Richter (2012) where a rather general frame of future work is sketched.

\section{Appendix}

Table 1 gives a brief comparison of norms and antinorms at the hand of the functional $|\cdot|_{q}, q \in R$. Note that $N_{q}=\left\{x \in \mathbb{R}^{n}:|x|_{q}=0\right\}$ and $H_{i}=$ $\left\{\left(x_{1}, \ldots, x_{n}\right)^{T} \in \mathbb{R}^{n}: x_{i}=0\right\}, i=1, \ldots, n$ are hyper planes in $\mathbb{R}^{n}$.

Table 1 Norms and antinorms

\begin{tabular}{lllll}
\hline Parameter & $q$ & $\in[1, \infty)$ & $\in(0,1]$ & $\in(-\infty, 0)$ \\
\hline Functional & $\mid . l_{q}$ & Norm & Antinorm & Semi-antinorm, $z \neq 0$ \\
Unit ball & $B_{q}$ & Convex & Radially concave & Radially concave \\
& & & W.r.t. standard fan in $\mathbb{R}^{n}$ & W.r.t. standard fan in $\mathbb{R}^{n}$ \\
Inverse image of 0 & $N_{q}$ & $\{0\}$ & $\{0\}$ & $\cup_{1}^{d} H_{i}$ \\
\hline
\end{tabular}


Table 2 Quantiles $\chi_{q}^{p}(n), p=3.3$

\begin{tabular}{lllllllllll}
\hline $\mathrm{q} \backslash \mathrm{n}$ & 1 & 2 & \multicolumn{1}{c}{3} & \multicolumn{1}{l}{4} & \multicolumn{1}{c}{5} & 10 & 15 & \multicolumn{1}{c}{20} & \multicolumn{1}{c}{25} & \multicolumn{1}{c}{30} \\
\hline 0.005 & 0.00 & 0.00 & 0.01 & 0.05 & 0.12 & 1.14 & 2.92 & 5.17 & 7.73 & 10.51 \\
0.01 & 0.00 & 0.00 & 0.02 & 0.08 & 0.20 & 1.47 & 3.51 & 6.00 & 8.77 & 11.76 \\
0.025 & 0.00 & 0.01 & 0.06 & 0.18 & 0.37 & 2.08 & 4.54 & 7.39 & 10.49 & 13.78 \\
0.05 & 0.00 & 0.02 & 0.12 & 0.32 & 0.60 & 2.75 & 5.58 & 8.75 & 12.15 & 15.71 \\
0.1 & 0.00 & 0.06 & 0.26 & 0.58 & 0.99 & 3.70 & 6.98 & 10.55 & 14.29 & 18.16 \\
0.9 & 2.95 & 5.19 & 7.07 & 8.78 & 10.39 & 17.70 & 24.42 & 30.86 & 37.12 & 43.25 \\
0.95 & 4.56 & 7.17 & 9.30 & 11.20 & 12.98 & 20.93 & 28.13 & 34.96 & 41.57 & 48.02 \\
0.975 & 6.31 & 9.21 & 11.53 & 13.60 & 15.52 & 24.00 & 31.61 & 38.79 & 45.70 & 52.42 \\
0.99 & 8.75 & 11.96 & 14.50 & 16.75 & 18.82 & 27.91 & 35.98 & 43.55 & 50.81 & 57.85 \\
0.995 & 10.67 & 14.07 & 16.75 & 19.11 & 21.29 & 30.78 & 39.16 & 47.00 & 54.49 & 61.74 \\
\hline
\end{tabular}

Table 3 Quantiles $\chi_{q}^{p}(n), p=2.5$

\begin{tabular}{lllllllllll}
\hline $\mathrm{q} \backslash \mathrm{n}$ & 1 & 2 & 3 & 4 & 5 & 10 & 15 & 20 & \multicolumn{1}{l}{25} & 30 \\
\hline 0.005 & 0.00 & 0.00 & 0.03 & 0.12 & 0.26 & 1.68 & 3.84 & 6.43 & 9.29 & 12.36 \\
0.01 & 0.00 & 0.01 & 0.06 & 0.18 & 0.37 & 2.06 & 4.46 & 7.27 & 10.33 & 13.57 \\
0.025 & 0.00 & 0.02 & 0.13 & 0.33 & 0.61 & 2.72 & 5.50 & 8.63 & 11.99 & 15.50 \\
0.05 & 0.00 & 0.05 & 0.23 & 0.52 & 0.89 & 3.42 & 6.53 & 9.95 & 13.56 & 17.31 \\
0.1 & 0.01 & 0.13 & 0.43 & 0.84 & 1.33 & 4.36 & 7.88 & 11.64 & 15.55 & 19.57 \\
0.9 & 2.83 & 4.86 & 6.60 & 8.20 & 9.72 & 16.70 & 23.19 & 29.43 & 35.51 & 41.50 \\
0.95 & 4.15 & 6.49 & 8.43 & 10.20 & 11.86 & 19.38 & 26.28 & 32.87 & 39.26 & 45.52 \\
0.975 & 5.56 & 8.13 & 10.24 & 12.15 & 13.93 & 21.92 & 29.17 & 36.06 & 42.71 & 49.21 \\
0.99 & 7.50 & 10.32 & 12.62 & 14.68 & 16.60 & 25.11 & 32.77 & 40.00 & 46.96 & 53.72 \\
0.995 & 9.01 & 12.00 & 14.41 & 16.57 & 18.58 & 27.44 & 35.37 & 42.83 & 50.00 & 56.95 \\
\hline
\end{tabular}

Table 4 Quantiles $\chi_{q}^{p}(n), p=0.75$

\begin{tabular}{lllllllllll}
\hline $\mathrm{q} \backslash \mathrm{n}$ & 1 & 2 & 3 & \multicolumn{1}{l}{4} & \multicolumn{1}{c}{5} & 10 & 15 & 20 & 25 & 30 \\
\hline 0.005 & 0.02 & 0.19 & 0.50 & 0.92 & 1.40 & 4.35 & 7.76 & 11.43 & 15.25 & 19.19 \\
0.01 & 0.03 & 0.25 & 0.62 & 1.08 & 1.61 & 4.74 & 8.31 & 12.10 & 16.04 & 20.08 \\
0.025 & 0.06 & 0.36 & 0.82 & 1.36 & 1.96 & 5.37 & 9.16 & 13.14 & 17.24 & 21.43 \\
0.05 & 0.10 & 0.49 & 1.02 & 1.64 & 2.29 & 5.96 & 9.94 & 14.08 & 18.33 & 22.65 \\
0.1 & 0.17 & 0.68 & 1.31 & 2.00 & 2.73 & 6.69 & 10.89 & 15.22 & 19.64 & 24.10 \\
0.9 & 2.14 & 3.64 & 5.01 & 6.32 & 7.59 & 13.63 & 19.43 & 25.10 & 30.68 & 36.22 \\
0.95 & 2.71 & 4.34 & 5.82 & 7.21 & 8.55 & 14.89 & 20.91 & 26.77 & 32.52 & 38.20 \\
0.975 & 3.27 & 5.02 & 6.58 & 8.04 & 9.45 & 16.04 & 22.25 & 28.27 & 34.17 & 39.99 \\
0.99 & 4.00 & 5.88 & 7.53 & 9.08 & 10.57 & 17.45 & 23.88 & 30.09 & 36.16 & 42.12 \\
0.995 & 4.55 & 6.51 & 8.23 & 9.84 & 11.37 & 18.45 & 25.04 & 31.38 & 37.55 & 43.62 \\
\hline
\end{tabular}

Table 5 Quantiles $\chi_{q}^{p}(n), p=0.5$

\begin{tabular}{lllllllllll}
\hline $\mathrm{q} \backslash \mathrm{n}$ & 1 & 2 & 3 & \multicolumn{1}{l}{4} & \multicolumn{1}{c}{5} & 10 & \multicolumn{1}{c}{15} & \multicolumn{1}{l}{20} & \multicolumn{1}{l}{25} & 30 \\
\hline 0.005 & 0.05 & 0.34 & 0.77 & 1.29 & 1.86 & 5.18 & 8.88 & 12.79 & 16.83 & 20.96 \\
0.01 & 0.07 & 0.41 & 0.89 & 1.45 & 2.07 & 5.54 & 9.37 & 13.39 & 17.52 & 21.73 \\
0.025 & 0.12 & 0.54 & 1.10 & 1.73 & 2.40 & 6.11 & 10.12 & 14.29 & 18.56 & 22.89 \\
0.05 & 0.18 & 0.68 & 1.31 & 1.99 & 2.71 & 6.63 & 10.80 & 15.10 & 19.48 & 23.93 \\
0.1 & 0.27 & 0.87 & 1.58 & 2.33 & 3.11 & 7.26 & 11.61 & 16.07 & 20.59 & 25.16 \\
0.9 & 1.94 & 3.34 & 4.64 & 5.89 & 7.10 & 12.95 & 18.60 & 24.14 & 29.62 & 35.06 \\
0.95 & 2.37 & 3.88 & 5.26 & 6.57 & 7.85 & 13.94 & 19.77 & 25.47 & 31.09 & 36.64 \\
0.975 & 2.79 & 4.38 & 5.83 & 7.21 & 8.54 & 14.84 & 20.82 & 26.66 & 32.39 & 38.05 \\
0.99 & 3.32 & 5.02 & 6.55 & 8.0 & 9.39 & 15.92 & 22.09 & 28.08 & 33.95 & 39.74 \\
0.995 & 3.72 & 5.49 & 7.07 & 8.57 & 10.0 & 16.69 & 22.99 & 29.08 & 35.04 & 40.91 \\
\hline
\end{tabular}


Competing interests

The author declares that he has no competing interests.

Acknowledgement

The author is thankful to the reviewers for their valuable hints leading to an improvement of the paper.

Received: 6 October 2015 Accepted: 14 March 2016

Published online: 01 April 2016

\section{References}

Arellano-Valle, RB, Richter, W-D: On skewed continuous $l_{n, p}$-symmetric distributions. Chil. J. Stat. 3(2), 193-212 (2012)

Arnold, BC, Castillo, E, Sarabia, JM: Multivariate distributions defined in terms of contours. J. Stat. Planning Inf. 138, 4158-4171 (2008)

Bolboaca, SD, Jäntschi, L, Sestras, AF, Sestras, RE, Pamfil, DC: Rearson-Fisher Chi-Square revisited. Information. 2, 528-545 (2011)

Bomsdorf, E: Induktive Statistik. Verlag Josef Eul, Köln (1989)

Burago, D, Burago, Y, Ivanov, S: A course in metric geometry. Graduate studies in mathematics, vol. 33. A.M.S., Providence RI (2001)

Fang, K-T, Kotz, S, Ng, K-W: Symmetric multivariate and related distributions. Chapman and Hall, London (1990)

Fernández, C, Osiewalski, J, Steel, MFJ: Modeling and inference with v-spherical distributions. JASA. 90(432), 1331-1340 (1995)

Fisher, RA: The conditions under which $x^{2}$ measures the discrepancy between observation and hypothesis. J. R. Stat. Soc 87, 442-444 (1924a)

Fisher, RA: On a distribution yielding the error functions of several well known statistics. Proc. Int. Cong. Math. Toronto. 2 , 805-813 (1924b)

Fisher, RA: Statistical methods for research workers. In: Classics in the History of Psychology. Internet Resource by C.D. Green, York University, Toronto, (1925). ISBN 0-05-002170-2, http://psychclassics.yorku.ca/Fisher/Methods. Accessed 23 Mar 2016

Haidenhain, J: Machining accuracy of machine tools. Technical information 635 399-22.5 (2011). www.heidenhain.de/ de_EN/php/documentation-information/brochures/popup/media/media/file/view/file-0209/file.pdf. Accessed 23 Mar 2016

Helmert, PR: Über die Wahrscheinlichkeit von Potenzsummen der Beobachtungsfehler etc. Z.f. Math. u. Phys. 21, 102-219 (1876)

Hlubinka, D, Vencalek, O: Depth-based classification for distributions with nonconvex support. J. Probab. Statist. 2013(629184), 7 (2013)

Ittrich, C, Krause, D, Richter, W-D: Probabilities and large quantiles of noncentral generalized Chi-square distributions. Statistics. 34, 53-101 (2000)

Kotz, S, Kozubowski, TJ, Podgórski, K: The Laplace distribution and generalizations. Birkhäuser, Boston (2001)

Livadiotis, G: Approach to general methods for fitting and their sensitivity. Physica A. 375, 518-536 (2007)

Livadiotis, G: Expectation values and variance based on $L^{p}$ norms. Entropy. 14, 2375-2396 (2012)

Livadiotis, G: Chi-p distribution: characterization of the goodness of the fitting using $L^{p}$ norms. J. Statist. Distr. Appl. 1, 4 (2014)

Mirvaliev, M: The components of chi-squared statistics for goodness of fit tests. J. Sov Math. 38, 2357-2363 (1987)

Moszyńska, M, Richter, W-D: Reverse triangle inequality. Antinorms and semi-antinorms. Studia Scientiarum Mathematicarum Hungarica. 49(1), 120-138 (2012)

Müller, K, Richter, W-D: Extreme value distributions for dependent jointly $l_{n, p}$-symmetrically distributed random variables. Depend. Model. 4, 30-62 (2016)

Pearson, $\mathrm{K}$ : On the criterion that a given system of deviations from the probable in the cases of a correlated system of variables is such that it can be reasonably supposed to have arisen from random sampling. Phil. Mag. 50, 157 (1900)

Pisier, G: The volume of convex bodies and Banach space geometry. University Press, Cambridge (1989)

Plackett, RL: Karl Pearson and the Chi-squared test. Int. Statst. Rev. 51, 59-72 (1983)

Richter, W-D: Laplace-Gauß integrals, Gaussian measure asymptotic behaviour and probabilities of moderate deviations. Z. Anal. Anw. 4(3), 257-267 (1985)

Richter, W-D: A geometric method in stochastics (in German). Rostock. Math. Kolloqu. 44, 63-72 (1991)

Richter, W-D: Generalized spherical and simplicial coordinates. J. Math. Anal. Appl. 336, 1187-1202 (2007)

Richter, W-D: Continuous $l_{n, p}$-symmetric distributions. Lith. Math. J. 49(1), 93-108 (2009)

Richter, W-D: Circle numbers for star discs. ISRN Geometry. 16(Article ID 479262), 16 (2011)

Richter, W-D: Exact distributions under non-standard model assumptions. AIP Conf. Proc. 1479, $442-445$ (2012). doi:10.1063/1.4756160

Richter, W-D: Geometric and stochastic representations for elliptically contoured distributions. Comm. Stat. Theory Methods. 42, 579-602 (2013)

Richter, W-D: Geometric disintegration and star-shaped distributions. J. Stat. Distributions Appl. 1, 20 (2014). doi:10.1186/s40488-014-0020-6

Richter, W-D: Star-shaped distributions: Euclidean and non-Euclidean representations. In: Proceedings of the 60th World Statistics Congress 2015. Rio de Janeiro, (2016). Internat. Statist. Inst., https://www.isi-web.org. Accessed 23 Mar 2016

Richter, W-D: Norm contoured distributions in $\mathbb{R}^{2}$. Lecture Notes of Seminario Interdisciplinare di Mathematica University di Basilicata Italy. 12, 179-199 (2015b)

Richter, W-D: Convex and radially concave contoured distributions. Probab. Stat. 12(Article ID 165468), 12 (2015c)

Richter, W-D: Representing continuous star-shaped probability measures in spaces with suitably constructed geometries. AIP Conf. Proc (2016). Accepted for print

Statlect: The digital textbook (2016). http://www.statlect.com/hypothesis_testing_variance.htm. Accessed 23 Mar 2016

Wilcox, R: Comparing the variances of two dependent variables. J. Stat. Distr. Appl. 2, 7 (2015) 